\title{
Semi-intrusive quantification of uncertainties in stochastic electromagnetic interactions: Analysis of a spectral formulation
}

\section{Citation for published version (APA):}

Sy, O. O., Beurden, van, M. C., Michielsen, B. L., \& Tijhuis, A. G. (2009). Semi-intrusive quantification of uncertainties in stochastic electromagnetic interactions: Analysis of a spectral formulation. In Proceedings of 11th international conference on Electromagnetics in Advanced Applications (ICEAA '09), 14-18 September 2009, Turin, Italy (pp. 552-555). Institute of Electrical and Electronics Engineers.

https://doi.org/10.1109/ICEAA.2009.5297372

DOI:

10.1109/ICEAA.2009.5297372

Document status and date:

Published: 01/01/2009

\section{Document Version:}

Publisher's PDF, also known as Version of Record (includes final page, issue and volume numbers)

\section{Please check the document version of this publication:}

- A submitted manuscript is the version of the article upon submission and before peer-review. There can be important differences between the submitted version and the official published version of record. People interested in the research are advised to contact the author for the final version of the publication, or visit the $\mathrm{DOI}$ to the publisher's website.

- The final author version and the galley proof are versions of the publication after peer review.

- The final published version features the final layout of the paper including the volume, issue and page numbers.

Link to publication

\section{General rights}

Copyright and moral rights for the publications made accessible in the public portal are retained by the authors and/or other copyright owners and it is a condition of accessing publications that users recognise and abide by the legal requirements associated with these rights.

- Users may download and print one copy of any publication from the public portal for the purpose of private study or research.

- You may not further distribute the material or use it for any profit-making activity or commercial gain

- You may freely distribute the URL identifying the publication in the public portal.

If the publication is distributed under the terms of Article 25fa of the Dutch Copyright Act, indicated by the "Taverne" license above, please follow below link for the End User Agreement:

www.tue.nl/taverne

Take down policy

If you believe that this document breaches copyright please contact us at:

openaccess@tue.nl

providing details and we will investigate your claim. 


\section{Semi-intrusive quantification of uncertainties in stochastic electromagnetic interactions: Analysis of a spectral formulation}

\author{
O.O. Sy * M.C. van Beurden*
}

\begin{abstract}
A stochastic approach is presented to statistically characterise uncertainties in electromagnetic interactions. A spectral definition of the observables allows for the assessment of the effect of a randomly deformed device independent of the incident field.
\end{abstract}

\section{Introduction}

In computational electromagnetics, many deterministic algorithms have been developed to model the interaction between a material system and an incident field for antenna design, scattering, and electromagnetic compatibility (EMC) purposes. However, in practice, the knowledge of the actual configuration can be affected by uncertainties concerning the features of the scatterer or the incident field, which can lead to significant deviations between simulations and measurements.

In these cases, rather than aiming for an exhaustive study of all the possible situations, which can be extremely tedious, stochastic rationales postulate that the unknown parameters of the configuration vary randomly. Probability theory is then employed to propagate this initial randomness through the model and to measure the randomness induced on its output, or observable, via statistical moments, that can be determined by a limited number of computations.

The propagation of the randomness can be performed non-intrusively by viewing the deterministic model as a black box, having the

\footnotetext{
*Department of Electrical Engineering, Eindhoven University of Technology, Den Dolech 2, 5600 MB, Eindhoven, The Netherlands, e-mail o.o.sy@tue.nl, m.c.v.beurden@tue.nl, a.g.tijhuis@tue.nl, tel.: +31 402474791.

†ONERA - DEMR, BP 74025, 2, av. Edouard Belin, 31055 Toulouse Cedex 4, France, e-mail: bastiaan.michielsen@onera.fr
}

\author{
B.L.Michielsen ${ }^{\dagger} \quad$ A.G. Tijhuis ${ }^{*}$
}

scattering device and the incident field as input, and providing the value of observables such as the induced voltage or current at a given port region $[1,2]$. However, evaluating the effect of a given geometrical randomness on different excitations requires as many statistical studies of the entire configuration, as there are incident fields. Alternatively, a semi-intrusive approach can be adopted to characterize the randomness of the receiving device independent of the incident field. This strategy has been applied by Michielsen in [3] with the aid of a Taylor expansion of the incident field, and by Brown in [4], where asymptotic relations are employed to simplify the formulation of the problem in the presence of an unbounded rough surface.

This paper proposes a semi-intrusive method based on a spectral reformulation of the observable, chosen as the voltage $V_{e}$ induced at the port of a receiving device. This spectral definition is introduced in Section 2. The deterministic model is randomized in Section 3 to express the average and the variance of $V_{e}$ in terms of the statistical moments of a current distribution depending solely on the scatterer. Results are then provided in Section 4 for the example of a transversely undulating wire.

\section{Deterministic parametrization}

A device enclosed in the perfectly conducting surface $S_{\alpha}$ and containing a port is considered. The geometry of $S_{\boldsymbol{\alpha}}$ is explicitly described and controlled by a vector $\boldsymbol{\alpha}=\left(\alpha_{1}, \ldots, \alpha_{n}\right)$, which belongs to the fixed domain $\mathcal{A} \subset \mathbb{R}^{n}$. External electromagnetic sources radiate the field $\boldsymbol{E}^{i}$, which induces the voltage $V_{e}$ at the port, i.e.

$$
V_{e}=-\int_{\boldsymbol{r} \in S_{\boldsymbol{\alpha}}} \boldsymbol{J}_{\boldsymbol{\alpha}}(\boldsymbol{r}) \cdot \boldsymbol{E}^{i}(\boldsymbol{r})
$$


The normalized distribution $\boldsymbol{J}_{\boldsymbol{\alpha}}$ depends solely on $S_{\boldsymbol{\alpha}}$ as it is induced, in the absence of $\boldsymbol{E}^{i}$, by a unit current source applied to the port. The solution of a frequency-domain electric-field integral equation to obtain $\boldsymbol{J}_{\boldsymbol{\alpha}}$ is the major numerical effort involved in this model.

Geometrical modifications of the setup are obtained by varying $\boldsymbol{\alpha}$ in $\mathcal{A}$, which in turn infers changes in $\boldsymbol{J}_{\boldsymbol{\alpha}}$ and in $V_{e}$. Further, although $\boldsymbol{E}^{i}$ is assumed to be independent of the scattering device, it still needs to be evaluated at points $\boldsymbol{r} \in S_{\boldsymbol{\alpha}}$, thereby complicating the task of characterizing the variations of $S_{\boldsymbol{\alpha}}$ independent of $\boldsymbol{E}^{i}$. This limitation is sidestepped by applying Plancherel's theorem [5, p. 186] to recast Equation (1) in

$$
V_{e}=-\int_{\boldsymbol{k} \in \mathbb{R}^{3}} \widetilde{\boldsymbol{J}_{\boldsymbol{\alpha}}}(\boldsymbol{k}) \cdot \widetilde{\boldsymbol{E}^{i}}(-\boldsymbol{k}),
$$

where $\widetilde{\boldsymbol{J}_{\boldsymbol{\alpha}}}$ and $\widetilde{\boldsymbol{E}^{i}}$ are the Fourier transforms, with respect to $\boldsymbol{r}$, of $\boldsymbol{J}_{\boldsymbol{\alpha}}$ and $\boldsymbol{E}^{i}$ respectively. The key advantage of Equation (2) lies in the fact that the effect of the randomness of $S_{\alpha}$ is entirely encompassed in $\widetilde{\boldsymbol{J}_{\boldsymbol{\alpha}}}$. The computation of $\widetilde{\boldsymbol{J}_{\boldsymbol{\alpha}}}$ is eased by the bounded support of $\boldsymbol{J}_{\boldsymbol{\alpha}}$, viz $S_{\boldsymbol{\alpha}}$. Further, the evaluation of the improper integral in Eq. (2) can be simplified by making assumptions on the type of excitation $\boldsymbol{E}^{i}$.

Given a finite set of fixed directions of incidence $\left\{\boldsymbol{u}_{1}, \ldots, \boldsymbol{u}_{M}\right\}$, together with the wavevectors $\mathcal{K}_{M}=\left\{\boldsymbol{k}_{l}=(2 \pi / \lambda) \boldsymbol{u}_{l}, l=1, \ldots, M\right\}$, where $\lambda$ denotes the wavelength, $\boldsymbol{E}^{i}$ can be constructed as the superposition of plane waves propagating along the directions $\mathcal{K}_{M}$ as follows

$$
\boldsymbol{E}^{i}: \mathbb{R}^{3} \ni \boldsymbol{r} \longmapsto \sum_{l=1}^{M} \boldsymbol{e}_{l} \exp \left(-j \boldsymbol{k}_{l} \cdot \boldsymbol{r}\right) \in \mathbb{C}^{3} .
$$

The polarization vectors $\left\{\boldsymbol{e}_{l} \in \mathbb{R}^{3}, \boldsymbol{e}_{l} \cdot \boldsymbol{k}_{l}=\right.$ $0, l=1, \ldots, M\}$ of the plane waves represent a degree of freedom allowing for the construction of a variety of incident fields. The contribution of a given direction $\boldsymbol{k}_{l}$ can, for instance, be "switched off" simply by setting $\boldsymbol{e}_{l}=\mathbf{0}$. Conversely, a single plane wave propagating in the direction $\boldsymbol{k}_{l} \in \mathcal{K}_{M}$ is obtained by canceling all the but the $l$-th polarization vector.
Interestingly, the Fourier counterpart of $\boldsymbol{E}^{i}$ becomes a combination of Dirac distributions and $\widetilde{\boldsymbol{J}_{\boldsymbol{\alpha}}}$ needs to be evaluated only for $\boldsymbol{k} \in \mathcal{K}_{M}$ to deduce the voltage $V_{e}$ as follows

$$
V_{e}(\boldsymbol{\alpha})=-\sum_{l=1}^{M} \widetilde{\boldsymbol{J}_{\boldsymbol{\alpha}}}\left(\boldsymbol{k}_{l}\right) \cdot \boldsymbol{e}_{l}
$$

A duality exists between the Eqs (1) and (4): in the spatial domain, the support of $\boldsymbol{J}_{\boldsymbol{\alpha}}$, viz $S_{\boldsymbol{\alpha}}$, determines the points at which $\boldsymbol{E}_{\boldsymbol{\beta}}^{i}$ is evaluated, whereas in the spectral domain, the support of $\widetilde{\boldsymbol{E}_{\boldsymbol{\beta}}^{i}}$, viz $\mathcal{K}_{M}$, dictates the directions in which $\widetilde{\boldsymbol{J}_{\boldsymbol{\alpha}}}$ is calculated.

\section{Randomization}

The effect of uncertainties concerning the geometry of $S_{\boldsymbol{\alpha}}$ is now investigated. To begin with, the variations of $\boldsymbol{\alpha}$ in $\mathcal{A}$ are assumed to be random according to a known probability density function $f_{\boldsymbol{\alpha}}$, which is chosen a priori. In a non-intrusive approach, the statistical moments of $V_{e}$, such as its mean are obtained as

$$
\mathbb{E}\left[V_{e}\right]=\int_{\boldsymbol{\alpha}^{\prime} \in \mathcal{A}} V_{e}\left(\boldsymbol{\alpha}^{\prime}\right) f_{\boldsymbol{\alpha}}\left(\boldsymbol{\alpha}^{\prime}\right) d \boldsymbol{\alpha}^{\prime},
$$

where the integral is approximated by a quadrature rule adapted to the dimension of $\mathcal{A}[2]$. On the other hand, with the representation of Equation (4) the randomness of $\boldsymbol{\alpha}$ will affect $V_{e}$ only via $\widetilde{\boldsymbol{J}_{\boldsymbol{\alpha}}}$, thereby enabling the definition of the mean and the variance of $V_{e}$ in terms of the average and the covariance of $\widetilde{\boldsymbol{J}_{\boldsymbol{\alpha}}}$,

$$
\mathbb{E}\left[V_{e}\right]=-\sum_{l=1}^{M} \mathbb{E}\left[\widetilde{\boldsymbol{J}_{\boldsymbol{\alpha}}}\right]\left(\boldsymbol{k}_{l}\right) \cdot \boldsymbol{e}_{l}
$$

$$
\operatorname{var}\left[V_{e}\right]=\sum_{l_{1}=1}^{M} \sum_{l_{2}=1}^{M} \boldsymbol{e}_{l_{1}} \cdot \mathcal{C}\left[\widetilde{\boldsymbol{J}_{\boldsymbol{\alpha}}}\right]\left(\boldsymbol{k}_{l_{1}}, \boldsymbol{k}_{l_{2}}\right) \cdot \boldsymbol{e}_{l_{2}}^{t},
$$

in which the superscript " $\mathrm{t}$ " indicates the vectorial transposition. Due to the fact that $\widetilde{\boldsymbol{J}_{\boldsymbol{\alpha}}}=$ $\left(\widetilde{J}_{\boldsymbol{\alpha}}^{x},{\widetilde{J_{\boldsymbol{\alpha}}}}^{y},{\widetilde{J_{\boldsymbol{\alpha}}}}^{z}\right)$ is a complex-valued vector, the covariance matrix $\mathcal{C}\left[\widetilde{\boldsymbol{J}_{\alpha}}\right]$ will be Hermitian and consist of $3 \times 3$ sub-matrices assessing the mutual correlation between the components of $\widetilde{\boldsymbol{J}_{\boldsymbol{\alpha}}}$. 
The statistics of $\boldsymbol{J}_{\boldsymbol{\alpha}}$ are also defined as integrals similar to Equation (5), which are computed by a quadrature rule suited to the dimension of $\mathcal{A}$. These statistics of $\boldsymbol{J}_{\boldsymbol{\alpha}}$ need to be computed only once and can then be employed to deduce the average and the variance of the voltage induced by any excitation resulting from a combination of plane waves associated to $\mathcal{K}_{M}$.

\section{Results}

This semi-intrusive rationale is illustrated through the example of a perfectly electrically conducting (PEC) thin-wire structure, which can be regarded as a transmission line. As shown in Figure. 1, the wire lies over an infinite PEC plane to which it is connected via two vertical wires, one of which contains a port.

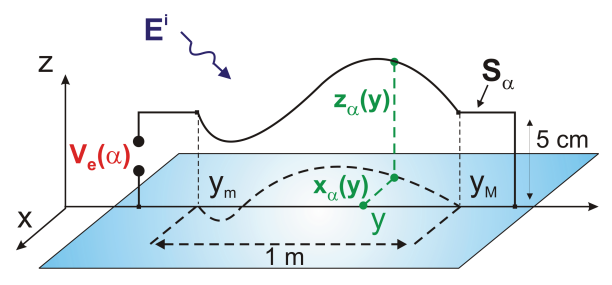

Figure 1: Thin-wire setup.

For any $y \in\left[y_{m}, y_{m}\right]$, the Cartesian coordinates of the axis correspond to

$$
\begin{aligned}
x_{\alpha}(y) & =\alpha_{1} \sin \left[2 \pi\left(y-y_{m}\right)\right] \\
z_{\alpha}(y) & =0.05+\alpha_{2} \sin \left[2 \pi\left(y-y_{m}\right)\right]
\end{aligned}
$$

where $\boldsymbol{\alpha}=\left(\alpha_{1}, \alpha_{2}\right)$ has statistically independent components that are uniformly distributed in $\mathcal{A}_{1}=\mathcal{A}_{2}=[-0.03,0.03] \mathrm{m}$. The frequency is chosen as $f=500 \mathrm{MHz}$, and the 50 directions of incidence $(M=50)$, from which the set $\mathcal{K}_{M}$ is obtained, are specified in polar coordinates by $\left\{\boldsymbol{u}_{l}: \theta_{i}(l)=l \pi /(2 M), \phi_{i}(l)=\pi / 4\right\}$ for $l=1, \ldots, M$.

The amplitude of the covariance matrix of $\widetilde{\boldsymbol{J}_{\boldsymbol{\alpha}}}$ is plotted in Figure 2. The symmetry in this figure results from the Hermitian nature of $\mathcal{C}\left[\widetilde{\boldsymbol{J}_{\boldsymbol{\alpha}}}\right]$. As mentioned in Section $3, \mathcal{C}\left[\widetilde{\boldsymbol{J}_{\boldsymbol{\alpha}}}\right]$ has a $3 \times 3$ structure where the sub-blocks indicate the mutual statistical correlation between the Cartesian components of $\widetilde{\boldsymbol{J}_{\boldsymbol{\alpha}}}$. Each sub-block expresses the intensity of the statistical correlation between the components of $\widetilde{\boldsymbol{J}_{\alpha}}$ for different directions of incidence in $\mathcal{K}_{M}$.

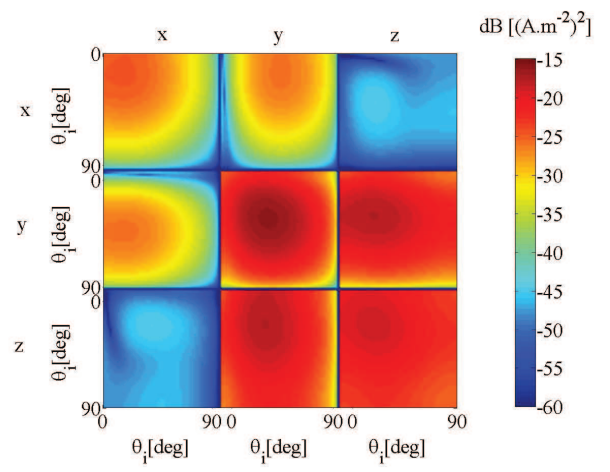

Figure 2: Amplitude of the covariance of $\widetilde{\boldsymbol{J}_{\alpha}}$ : $\mathcal{C}\left[\widetilde{\boldsymbol{J}_{\alpha}}\right]_{d B}\left(\boldsymbol{k}_{l_{1}}, \boldsymbol{k}_{l_{2}}\right)=10 \log \left(\left|\mathcal{C}\left[\widetilde{\boldsymbol{J}_{\alpha}}\right]\left(\boldsymbol{k}_{l_{1}}, \boldsymbol{k}_{l_{2}}\right)\right|\right)$.

The component ${\widetilde{J_{\boldsymbol{\alpha}}}}^{y}$ has the largest autocorrelation, with a maximum around $\theta_{i}=25^{\circ}$. This feature stems from the orientation of the wire mainly along the $y$ direction. Although the geometrical undulations of $S_{\boldsymbol{\alpha}}$ are identical in the $x$ and $z$ directions, the auto- and crosscorrelation terms involving ${\widetilde{J_{\alpha}}}^{z}$ outweigh those of ${\widetilde{J_{\alpha}}}^{x}$. This difference is due to the presence of the ground plane, which enhances the effect of the vertical geometrical variations. Further, the very low statistical coupling between $\widetilde{J}_{\boldsymbol{\alpha}}^{x}$ and $\widetilde{J_{\alpha}}{ }^{z}$ is worth mentioning. This lack of correlation can be related to the independence of $\alpha_{1}$ and $\alpha_{2}$, by using transmission-line theory.

The tensors $\mathbb{E}\left[\widetilde{\boldsymbol{J}_{\boldsymbol{\alpha}}}\right]$ and $\mathcal{C}\left[\widetilde{\boldsymbol{J}_{\boldsymbol{\alpha}}}\right]$ are now successively tested by $M$ plane waves, each one associated with a given wave vector in $\mathcal{K}_{M}$, with a unit amplitude and a $\theta$ polarization. For each of these plane wave, the mean and the variance of $V_{e}$ are computed both non-intrusively using Equation (5), and semi-intrusively via Equations (6) and (7). The results, displayed in Figure 3 highlight the match between both results. The module $\left|\mathbb{E}\left[V_{e}\right]\right|$ oscillates between $60 \mathrm{mV}$ and $260 \mathrm{mV}$ as $\theta_{i}$ varies in $\left[0^{\circ}, 90^{\circ}\right]$, whereas $\sigma\left[V_{e}\right]$ has a maximum value of $65 \mathrm{mV}$ 
for $\theta_{i}=25^{\circ}$, as expected from the analysis of Figure 2.

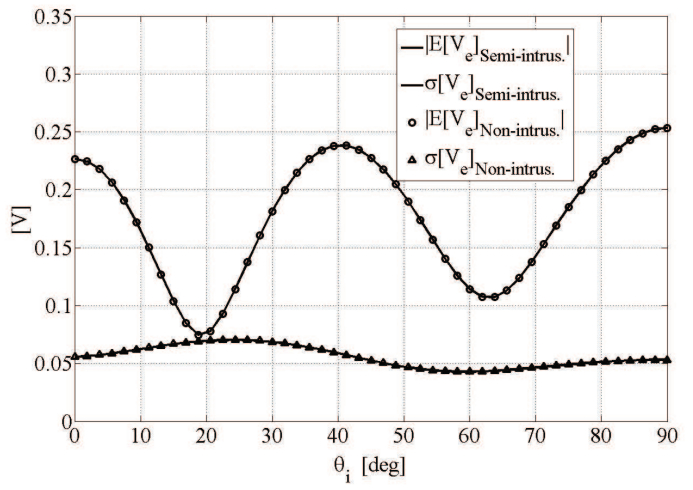

Figure 3: $\left|\mathbb{E}\left[V_{e}\right]\right|$ and $\sigma\left[V_{e}\right]=\sqrt{\operatorname{var}\left[V_{e}\right]}$ computed non-intrusively and a semi-intrusively.

The main asset of the semi-intrusive method appears when considering the cumulative computation time depicted in Figure 4. The precomputation of the statistical moments of $\widetilde{\boldsymbol{J}_{\alpha}}$ amounts to a duration of 49 seconds, after which the evaluations of $\mathbb{E}\left[V_{e}\right]$ and $\sigma\left[V_{e}\right]$ are performed very rapidly in $5 \mathrm{~ms}$. Comparatively, via the non-intrusive approach, for each incident field, the computation of $\mathbb{E}\left[V_{e}\right]$ and $\sigma\left[V_{e}\right]$ amounts to approximately 8 seconds.



Figure 4: Cumulative computation time.

\section{Conclusions}

The semi-intrusive approach presented in this paper hinges on a spectral definition of the ob- servables via Plancherel's theorem. The resulting representation allows for a refined statistical characterization of the randomness of a receiving device independent of the incident field. This feature is particularly appreciable in EMC when a given device needs to be tested under various types of excitations. Moreover, significant gains in computation time are achieved when multiple incident fields need to be considered, as demonstrated in the case of the thin wire. The semi-intrusive rationale also permits the study of random incident fields resulting from combinations of plane waves. The extension of this method to handle more general types of excitations is depends on the use of an efficient method to interpolate the spectrum of the incident field.

\section{Acknowledgements}

This work is funded by the Dutch Ministry of Economic Affairs, in the Innovation Research Program (IOP) number EMVT 04302.

\section{References}

[1] D. Bellan and S. Pignari, "Estimation of crosstalk in nonuniform cable bundles," in Proc. International Symposium on EMC, vol. 2, 2005, pp. 336-341.

[2] O. Sy, M. van Beurden, and B. Michielsen, "Analysis of stochastic resonances in electromagnetic couplings to transmission lines," in Proc. 20th International Zürich Symposium on EMC, 2009, pp. 33-36.

[3] B. L. Michielsen, "Probabilistic modelling of stochastic interactions between electromagnetic fields and systems," Comptes Rendus de l'Académie des sciences: Physique, vol. 7, pp. 543-559, 2006.

[4] G. S. Brown, "A Stochastic Fourier Transform Approach to scattering from perfectly conducting randomly rough surfaces," IEEE Trans. Ant. Prop., vol. AP-30, no. 6, pp. 1135-1144, 1982.

[5] W. Rudin, Real and complex analysis, 3rd ed. New York: McGraw-Hill, 1987. 